\title{
Design of Transmission Scheme for a Special Manipulator
}

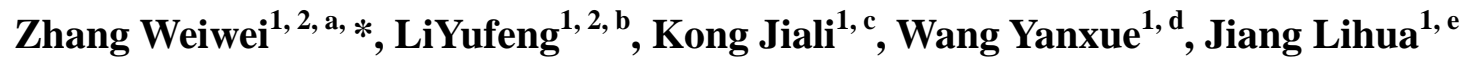 \\ ${ }^{1}$ School of Mechanical Engineering, Shandong Huayu University of Technology, Dezhou 253034, China \\ ${ }^{2}$ Dezhou Municipal Key Laboratory of Industrial Robot Control, Dezhou 253034, China \\ a zhang8412860@163.com, ${ }^{b}$ lyf@sdhyxy.com, ${ }^{c}$ kongjialiskd@126.com, ${ }^{\mathrm{d}}$ dzytwyx@126.com, ${ }^{\mathrm{j}}$ janglihuajian \\ g126@126.com \\ *corresponding author:zhang8412860@163.com
}

Keywords: manipulator, transmission scheme, motion control, four axis

\begin{abstract}
A kind of high automatic manipulator is designed, the joints of manipulator are controlled by servo motor. The manipulator is used for manufacturing lower cover of soybean milk machine. The manipulator includes four axis which are the upper and lower axis, the rotary axis, the telescopic axis and the arm rotating axis. The transmission scheme of the manipulator is confirmed. Simulation experiments prove that, the speed of the manipulator satisfies the requirements, and the motion is smooth, whose positioning is accurate and complicated.
\end{abstract}

\section{Introduction}

With the continuous development of modern manufacturing technology, the high-speed automation technology in stamping processing has gradually replaced the traditional production methods such as special machine operation and manual loading and unloading. Manipulator plays an important role in this process [1][2]. A four axis and double speed manipulator is studied in this paper. In the design of the manipulator, the design of the transmission scheme is one of the most important designs. The transmission form directly affects the overall layout of the manipulator, which is closely related to its speed, load and load bearing capacity. According to the actual requirements, the selection of appropriate transmission scheme is the first consideration in the mechanical and mechanical design [3]. Only the transmission scheme is determined first, and the following structure design and electrical design have some basis. In the mechanical design, there are several typical transmission modes, gear and rack drive, ball screw drive, synchronous wheel synchronous belt drive and so on. Different servo axes choose different transmission schemes.

\section{Design of Transmission Scheme of the Manipulator}

\subsection{Selection of Upper and Lower Shaft Transmission Scheme}

The I axis of the manipulator is the upper and lower drive shaft. It is the heaviest axle in the shaft of the whole manipulator, and the rest of the axes are moving on his foundation. I axis use sthe ball bearing screw transmission mode with strong bearing capacity, fast reaction speed and high positioning accuracy to complete the up and down reciprocating motion. The main components are: servo motor with brake, one to one synchronous belt, synchronous belt, precision screw support, precision ball screw, screw nut, linear guide and support plate, etc. The whole movement process is: the servo motor turns, passes the rotation movement through the synchronous belt and the synchronous wheel to the ball screw. The rotation of the ball screw drives the screw nut to do the upper and lower movement of the screw nut, thus driving the slide plate fixed to the nut to move up and down straight line, and complete the up and down movement of the manipulator. The upper and lower shaft transmission scheme is shown in Figure 1. 


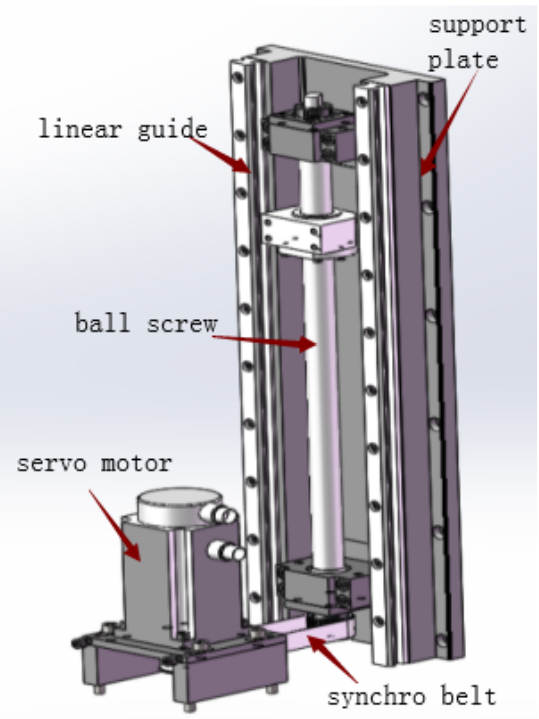

Figure 1 Transmission scheme of upper and lower shaft

\subsection{Selection of Rotating Axis Transmission Scheme}

The scheme used in the rotating shaft is that the servo motor uses one - to - one synchronous belt and pulley to drive the harmonic reducer to rotate, thus driving the III axis and the IV axis to rotate. The main part of this part is the choice of rotary reducer. Because of the high load weight, low speed and large inertia, the transmission ratio is large, the ordinary reducer cannot meet the requirements, the relatively harmonic reducer is selected. The transmission scheme of the rotating shaft is shown in Figure 2.

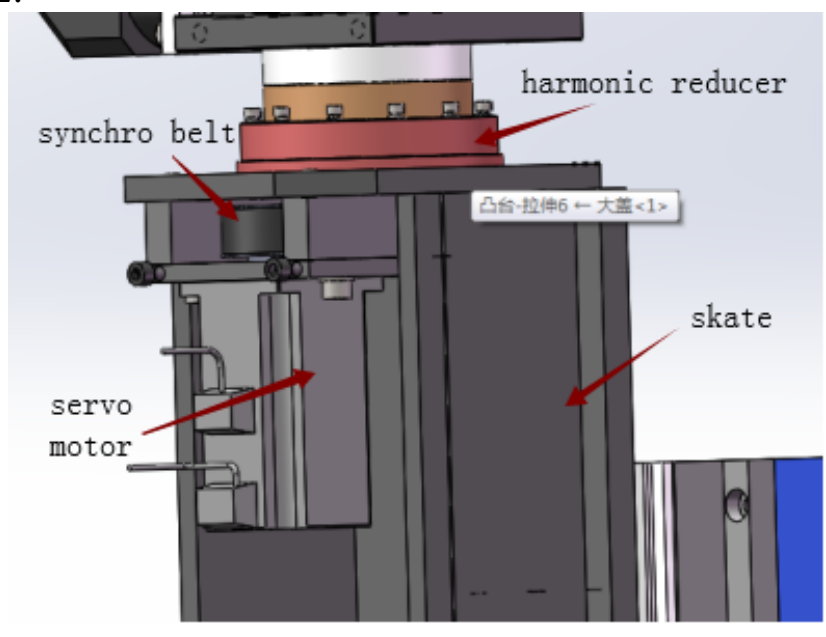

Figure 2 Transmission scheme of rotating shaft

\subsection{Selection of Telescopic Axis Transmission Scheme}

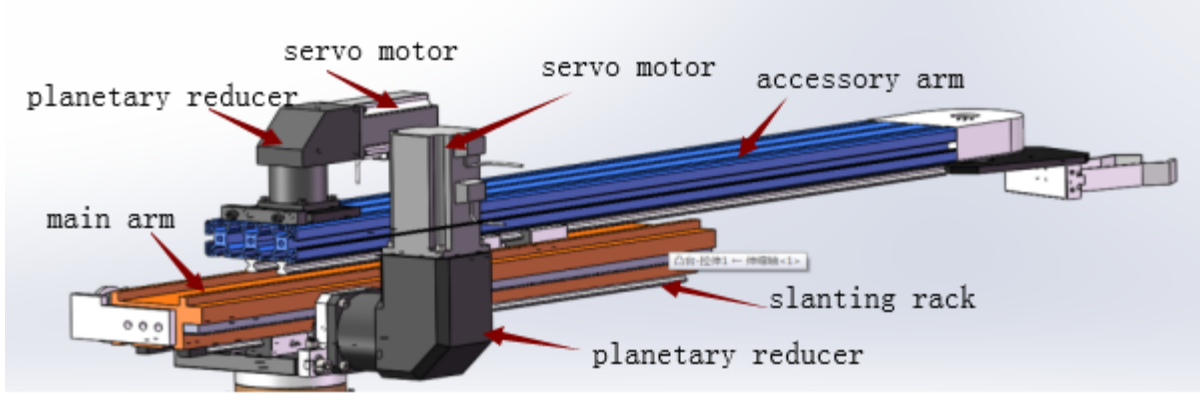

Figure 3 Transmission scheme of telescopic shaft

The telescopic shaft refers to the back axis, which directly defines the overall working radius of the manipulator. The carrying capacity of the whole manipulator is closely related to the design of 
the telescopic shaft. The flexibility and expansion speed of the manipulator are all more important in the whole design. The telescopic shaft of the design adopts double speed structure form, the servo motor drives the skew rack through the helical gear, the rack is fixed on the arm of the aluminum alloy, and the main arm is telescopic movement. The side of the main arm is equipped with synchronous belt and synchronous wheel mechanism [4]. One end of the synchronous belt is fixed on the base of the III axis and the other end is fixed at the other end. On the vice arm, when the main arm is moving, the speed of the arm will double as the main arm moves together, which is the main highlight of this manipulator design. Both the main arm and the auxiliary arm are made of aluminum profiles. The transmission plan of telescopic shaft is shown in Figure 3, double speed structure schematic diagram are shown in Figure 4.

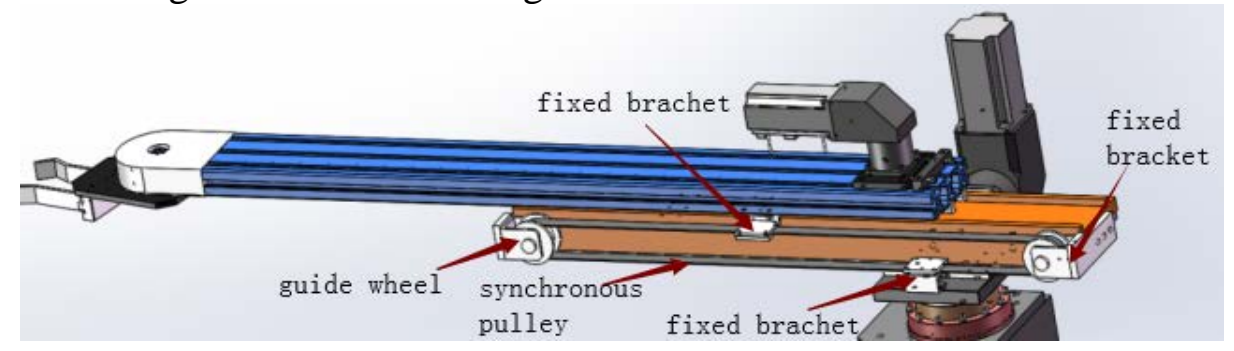

Figure 4 Double speed structure

\subsection{Selection of Transmission Scheme of Revolving Axis of the Hand}

The hand axle shaft is to grab the execution axis of the workpiece, the hand shaft is installed on the III axis, and the requirement is the rotary motion, because the main weight negative is the workpiece. The workpiece in this design is the lower cover of the soya bean milk machine. The quality is very light, so the bearing is not considered. The drive rotary servo electric machine is placed on the other end of the hand, so the electricity is placed. The distance between the machine and the paw is very far. The synchronous belt synchronous wheel transmission mode is used, the transmission mode can transmit torque long distance, and the reaction is fast and accurate [5]. The transmission scheme of the revolving shaft of the hand is shown in Figure 5.

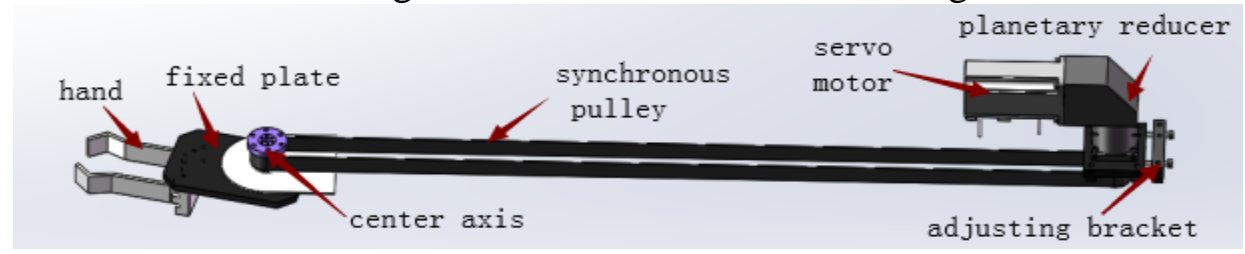

Figure 5 Transmission scheme of hand claw revolving shaft

\section{Experiments}

The mathematical model of the manipulator is established by using MATLAB software, and the space trajectory of the manipulator at two working points and the speed, displacement and acceleration curves of each degree of freedom joint are simulated. From the simulation curve, the manipulator is stable and reliable. The end gripper map space trajectory is shown in Figure 6.

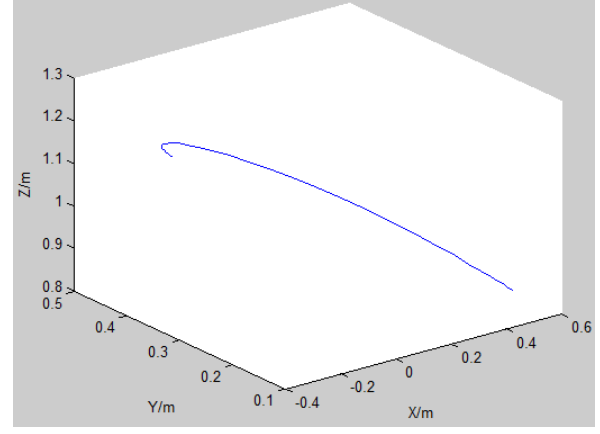

Figure 6 The space trajectory of end gripper 


\section{Conclusions}

The overall transmission plan of the manipulator is studied in this paper. The manipulator has four independent drive axes, that is, the four axis manipulator. It is mainly driven by the servo motor, and the ball screw pair, the gear rack and the synchronous belt wheel are used as the driving means. The reducer is used as the method of strengthening the power to determine the whole transmission scheme. Experiments prove that the manipulator react quickly, respond in time, with high positioning accuracy, reliability and convenient maintenance.

\section{References}

[1] Yu, Y. W. (2011). Development of economical automatic feeding manipulator for medium and small punch press[J]. Forging and Stamping Technology,36(6):66-69.

[2] Ye, Y. W. (2010). Research on rapid die changing technology of stamping equipment [J]. Mechanical manufacturing,48(5):70-73.

[3] Zhu, J. (2006). Design and analysis of manipulator for mobile robot [D]. Nanjing: Nanjing University of Science and Technology.

[4] H. Schaf, EkenelT. A robot learns to know people-first contacts of a robot Advance in Artificial Intelligence-29th Annual German Conference on AI, 2007(4):302-316.

[5] W Wang, M Lamb,IJ Busch-Vishniac. An automated loading and unloading system for a maglev wafer transport path[J]. Semiconductor Manufacturing, IEEE.Transaction.1993,6(3):276-279. 